Meta

Journal des traducteurs

Translators' Journal

\title{
En dépit du bon sens, le paradoxe du traducteur
}

André Chassigneux et Évelyne Clavaud

Volume 37, numéro 2, juin 1992

URI : https://id.erudit.org/iderudit/002223ar

DOI : https://doi.org/10.7202/002223ar

Aller au sommaire du numéro

Éditeur(s)

Les Presses de l'Université de Montréal

ISSN

0026-0452 (imprimé)

1492-1421 (numérique)

Découvrir la revue

Citer cet article

Chassigneux, A. \& Clavaud, É. (1992). En dépit du bon sens, le paradoxe du traducteur. Meta, 37(2), 204-213. https://doi.org/10.7202/002223ar d'utilisation que vous pouvez consulter en ligne.

https://apropos.erudit.org/fr/usagers/politique-dutilisation/ 


\title{
EN DÉPIT DU BON SENS, LE PARADOXE DU TRADUCTEUR
}

\author{
ANdré Chassigneux et ÉVElyne Clavaud
}

Université Paris $I I I$, Paris, France

Il est assis au bord de son fauteuil, à la fois aux aguets et tassé sur lui-même. Bernard Pivot se tourne enfin vers lui.

- J'avais invité le grand écrivain argentin José Cárcel Mayor pour nous parler de son nouveau roman, Souffler n'est pas jouer; mais, au dernier moment, il n'a pas obtenu son visa de sortie. J'ai donc fait appel à son traducteur attitré, Jérôme Lherer, qui a bien voulu venir à l'improviste et je l'en remercie. Je sais que je vous demande l'impossible, mais pouvez-vous résumer le sujet?

La caméra cadre le visage de Jérôme, angelot épanoui dans sa quarantaine. Un instant, le reflet d'un projecteur dore ses cheveux blonds. Il toussote et commence :

- Je pense qu'on peut parler du cheminement solitaire d'un homme à la recherche d'une certaine paix intérieure...

Sur l'écran (mais il ne le sait pas), sa tête est remplacée en fondu par la belle gueule aux traits burinés de Cárcel Mayor...

L'émission est terminée. Jérôme détache son micro-cravate, se lève et suit, à distance respectueuse, les autres invités qui se dirigent à petits pas cassés vers les rafraîchissements.

Les écrivains se congratulent, un verre de champagne à la main. Jérôme, lui, savoure son jus de fruit. Somme toute, il ne s'en est pas trop mal tiré...

-Quoi ? On traduit du Cárcel Mayor et on boit du jus d'orange? Non je plaisante... Quel ouvrage décapant, et comme vous l'avez bien défendu!

Jérôme fait face à la jeune femme qui s'adresse à lui.

- Je suis assez fier de l'épilogue, mais je dois dire que l'argot des pêcheurs...

- Mais... vous l'avez rencontré...

- Euh...

- Quelle personnalité fascinante! Racontez-moi tout...

- Je vais vous décevoir... Je ne l'ai, hélas, jamais rencontré.

La jeune femme prend congé aussi rapidement que la politesse le permet. Jérôme envisage de demander un second jus de fruit mais se ravise à temps : il n'a traduit que dix pages sur les vingt qu'il s'était fixées pour aujourd'hui.

Aux yeux d'un public généralement ignorant des langues étrangères, la capacité de comprendre ces mêmes langues semble un don magique qui confère à l'heureux élu un pouvoir quasi illimité. Il n'est pas rare que, dans une entreprise, on dépose sur le bureau d'une traductrice d'espagnol un texte écrit en chinois (nous voulons dire en mandarin) en lui disant: «Tiens, toi qui sais les langues...» Encore cette attitude témoigne-t-elle d'une grande ouverture d'esprit chez un patron qui a au moins le mérite de reconnaître la traduction comme un mal nécessaire. Il y a pire. Le don des langues étant considéré comme suffisant, on fait appel à un ingénieur maison qui a passé deux mois à New York, en partant du principe qu'il suffit de savoir faire tourner un moteur pour en parler intelligiblement, sinon intelligemment. Au nom de ce même principe (d'économie?), les scientifiques dits francophones rédigent directement en anglais des communications 
illisibles pour toute personne normalement constituée. Encore, si le traducteur n'était qu'inutile! Certains ne manquent pas de voir en lui un véritable danger public. C'est tellement facile de le rendre systématiquement responsable des incidents diplomatiques, de la perte de contrats à l'étranger, des mauvaises ventes de livres qui avaient pourtant fait de gros tirages dans leur pays d'origine. Et comme c'est tentant, quand on se targue de savoir l'anglais, d'accabler de sarcasmes le malheureux traducteur à l'occasion d'une faute particulièrement évidente...

Le traducteur, comme tous les hommes, doit assumer sa bêtise, mais lui, en plus, doit faire sienne celle des autres. On ne lui demande pas son avis sur la qualité des textes qu'on lui confie. Ainsi, le héros de La Nuit de Londres d'Henri Thomas : «un traducteur de dépêches d'agence et de documents officiels débite à longueur de journée un langage mort et stéréotypé dont, après trois mois, il connaît tous les tours et toutes les figures ${ }^{1} \gg$. A notre époque technico-commerciale, l'écrit est devenu le monopole de communicateurs professionnels qui gagnent leur vie en prenant les autres pour des imbéciles. Le traducteur est donc obligé de se spécialiser dans : a) le mode d'emploi rédigé à l'usage de gamins débiles et pervers ( 1 - ouvrir le carton en rabattant vers l'extérieur les deux panneaux du haut. 2 - sortir l'ordinateur en plaçant les mains de part et d'autre de l'appareil et l'amener progressivement vers le haut) ; b) le rapport en langue de bois (Il est fait pression sur la Maison Blanche pour qu'elle envoie un signal positif en donnant aux directives contre la discrimination frappant les fonctionnaires atteints du SIDA la stature d'une proclamation présidentielle = Reagan doit déclarer officiellement qu'il est contre la discrimination envers les fonctionnaires atteints du SIDA); c) le mémo tartuffesque (l'implantation du système informatique GASP va nous permettre de dynamiser notre structure à tous les niveaux = vous êtes virés).

Le mercredi venu, Jérôme commence à s'inquiéter: c'est demain que paraît $L e$ Monde des livres. On va sûrement parler du livre de Cárcel Mayor... mais va-t-on parler de $l u i$ ? Il a bon espoir ; tout le monde l'a vu à Apostrophes. Le critique le plus débordé aura bien trouvé une minute pour glisser une ligne sur la traduction. Il imagine déjà la formule «traduction qui respecte parfaitement le style décapant de Cárcel Mayor». Et, qui sait: «Jérôme Lherer ouvre la voie à une nouvelle race de traducteurs.» Mais si par hasard il lisait : «La traduction évacue complètement la flexuosité de l'original»? Jérôme préfère penser à autre chose. Machinalement, il attrape le dernier disque des Ponga-Tenga ${ }^{2}$, qu'il vient d'acheter. Au premier accord de guitare, il est déjà transporté dans ce village haut perché, il voit les enfants, sous le soleil torride, dévaler le flanc de la montagne à dos d'âne, pour se jeter, exténués, dans les égouts de la ville. Dans les égouts? Il a sursauté. Pourtant, alcantarilla signifie bien égout. C'est bien comme ça qu'il l'a traduit dans Souffler n' est pas jouer. Soudain, un doute... Il plonge dans le dictionnaire : alcantarilla: 1) égout... 5) (américanisme) réservoir (d'eau). C'est la faute qui ne pardonne pas. Pour moins que cela, des confrères ont été rayés des listes des éditeurs, réduits à traduire pour le reste de leurs jours des modes d'emploi de réveils thermo-dynamiques jetables. Que faire ? Téléphoner à l'éditeur pour qu'il imprime un erratum? Trop tard. Jérôme imagine déjà les sourires méprisants de son libraire...

Au même moment, un célèbre critique dont nous tairons le nom rentre chez lui, après un dîner bien arrosé, et aperçoit avec effroi le livre de Cárcel Mayor, qu'il n'a pas encore ouvert. Il n'a plus que quelques heures pour écrire son papier... Heureusement, il dispose d'une technique éprouvée pour faire face à ce genre de crise : une page sur vingt.

Le traducteur peut être tranquille: ni le critique, ni a fortiori le lecteur ne connaîtront l'original puisque la traduction a pour raison d'être de s'y substituer. Traduire, trahir, détruire, peut-être. C'est là qu'est l'obstacle. Ce texte que j'aime, ou que j'aimerais aimer, puisque je veux le faire connaître, je dois l'anéantir. L'original bien sûr 
existe toujours, mais pas pour celui qui me lira. Il n'en verra que le reflet nécessairement déformé que je lui offre. Le prisonnier, après sa permission, redescend dans la caverne, la tête et la bouche pleines de récits ensoleillés, mais il sait bien que s'il les raconte aux autres prisonniers qui ne sont jamais sortis, ils ne le croiront pas et au mieux se moqueront de lui, au pis chercheront à l'écharper. Il lui faut donc en estomper les teintes, en adoucir les contours pour rendre le soleil compréhensible à ceux qui ne connaissent que le feu artificiel. Il ne peut se faire accepter que s'il ment.

Ainsi du traducteur. Il doit faire passer sa propre pâleur pour le teint bronzé de l'écrivain, le français de Racine pour l'anglais de Shakespeare. C'est ici que le Tradutore, tradittore prend son véritable sens, et non dans le vieux cliché encore bien portant du traducteur traître au message de l'original. Oui, le traducteur trahit l'auteur puisqu'il prend sa place. Il trahit le texte puisqu'il le fait disparaître. Mais c'est là son paradoxe : plus il est fidèle, plus il trahit. En effet, plus sa traduction s'approche de l'original (encore faudrait-il définir «s'approcher»; contentons-nous de dire «s'approche de l'effet produit par le texte de départ»), plus elle se substitue à lui et plus elle rend inutile la lecture de cet original. Mais ce faisant, il se trahit lui-même. En tuant l'original, il se suicide. En effet, son métier le condamne à l'obscurité puisqu'il le prive automatiquement de toute louange - ou critique - qui pourrait découler d'une comparaison de sa traduction avec le texte de départ. Seules ses trahisons (au premier degré, c'est-à-dire ses bêtises) peuvent le faire reconnaître comme (mauvais) traducteur. Autrement dit, seules ses fautes peuvent témoigner de son existence. Il ne peut survivre, voire gagner sa vie, qu'en s'effaçant. En un mot, il ne brille que par son absence. Or, autre paradoxe, le traducteur revendique hautement cette attention de la critique et du public que précisément il ne peut obtenir qu'en trahissant l'original. Il est donc pris au piège d'un dilemme: soit il s'enferme dans une fidélité qui l'aveugle, soit il se fait reconnaître par ses erreurs.

Le traducteur n'est ni ange ni bête, et lui aussi fait la bête en voulant faire l'ange; ou plutôt, en voulant faire fidèle, il fait fi d'ailes. Il ronge tout ce qui pourrait l'emporter au-dessus des mots. Les plus grands poètes eux-mêmes se coupent parfois les ailes. Les traductions des contes d'Edgar Poe par Baudelaire sont, de l'avis même de certains anglophones, plus «fidèles» que l'original, parce qu'écrites dans une langue moins gothique et moins archaiisante que celle de Poe. Par ailleurs, Baudelaire est aussi capable de s'approprier des vers écrits en anglais, faisant vraiment œuvre de traducteur. Le Guignon, par exemple, est construit à partir d'un fragment de Longfellow (A Psalm of Life) et d'un autre de Gray (Elegy Written in a Country Churchyard) ${ }^{3}$.

Pour soulever un poids si lourd,

Sisyphe, il faudrait ton courage !

Bien qu'on ait du cœur à l'ouvrage,

l'Art est long et le Temps est court.

Loin des sépultures célèbres,

Vers un cimetière isolé,

Mon cour, comme un tambour voilé,

Va battant des marches funèbres.

Maint joyau dort enseveli

Dans les ténèbres et l'oubli,

Bien loin des pioches et des sondes.

Mainte fleur épanche à regret

Son parfum doux comme un secret

Dans les solitudes profondes. 
De ce poème, nous retiendrons simplement une trouvaille à faire pâlir de jalousie tous les traducteurs d'anglais: Baudelaire réussit l'exploit de rendre poétiquement en français la forme grammaticale du passé continu (are beating - va battant). On entend mieux battre son tambour que celui de Longfellow.

Or, le même Baudelaire, quand il traduit The Raven (Le Corbeau) de Poe, perd ses ailes par souci de fidélité. Ce poème est entièrement construit sur un nevermore (mot à mot : jamais plus) évoquant pour un lecteur anglais à la fois le cri du corbeau et la mort d'une jeune fille nommée Leonore. Baudelaire s'est cru obligé de faire un arbitrage entre la forme (en l'occurrence la sonorité des mots) et le sens (en l'occurrence la mort). Il a choisi de garder le sens (jamais plus) et de laisser perdre en français la forme, le son de cette même mort. Il aurait peut-être pu essayer de conserver les deux (par exemple avec «encore»). En s'en tenant à un sens littéral, il a créé cet être hybride, ce corbeau qui, au mépris de toutes les lois de l'ornithologie, croasse «jamais plus».

Comment expliquer que Baudelaire cesse d'être poète dès lors qu'il devient traducteur? C'est qu'il traduit Longfellow sans le faire exprès alors qu'il a conscience de traduire Poe - ce qui l'entrâine à se raccrocher au mot... pour se faire oublier.

Ce souci de se faire oublier, c'est ce que l'on entend généralement par objectivité. Ce culte de la neutralité, hélas courant à notre époque, vient encore d'inspirer des sacrifices. Une seule victime, mais de taille: Sigmund Freud. Écoutons André Bourguignon nous présenter la nouvelle traduction des ceuvres complètes. «Le but de l'entreprise, nous assure-t-il, est de mettre le lecteur français dans la même situation que le lecteur allemand devant le texte de Freud»; on s'efforcera donc de «respecter la syntaxe de Freud», ce qui nous permettra de «retrouver Freud, et pas un Freud francisét»».

Ainsi, les traducteurs des OCF auraient eu pour mission de mettre le lecteur français dans la même situation qu'un lecteur allemand. On ne peut qu'applaudir. Encore faudrait-il qu'on nous définît objectivement ladite situation. S'agit-il de mettre un lecteur français de 1991 dans la situation d'un lecteur allemand de 1900 ? Ou alors de mettre un lecteur français de 1991 dans la situation d'un lecteur allemand de 1991 ? Ou encore la combinaison des deux ? Le respect de la syntaxe de Freud doit-il aller jusqu'à obliger le lecteur français à marquer une pause pour mieux comprendre une phrase ambiguë, à l'endroit même où l'aurait fait le lecteur allemand de 1900 ou de 1991 ? En outre, le lecteur français devra-t-il assimiler une structure typiquement allemande dans l'espoir de pouvoir un jour savoir qu'il est enfin dans la même situation que le lecteur allemand de 1900 ou de 1991 ? Ainsi, combien de fois faudra-t-il lire «rétrofantasier» pour sentir peser sur ses épaules le poids d'un Zurück phantasieren ? Faudra-t-il à tout prix, toujours dans un souci d'objectivité, fabriquer un dérivé français sur le même modèle que le terme utilisé par Freud (Vergehen < gehen = passagèreté < passer) ? Et pourquoi pas l'allérité ? Si l'entreprise a réellement pour but de mettre le lecteur français dans la même situation que le lecteur allemand, pourquoi éprouve-t-on le besoin de la couronner par un glossaire franco-français? (Car on nous en promet un, comme s'il fallait se faire pardonner tant de néologismes barbares. Quel progrès! Freud lui-même n'y avait pas songé !)

Il faut se rendre à l'évidence: Freud a écrit en allemand. On n'y peut rien; lui non plus - c'était sa langue maternelle, celle de ses lecteurs. S'il avait cru faciliter la compréhension de son message par un glossaire, il en aurait rédigé un. Il ne l'a pas fait, donc on peut supposer qu'il employait une langue directement compréhensible par un «honnête homme». On peut aussi supposer qu'il ne s'amusait pas à disséquer sous microscope les mots de la langue allemande pour les recoller après $($ sehn + sucht $=$ sehnsucht). Il avait peut-être autre chose à donner au monde...

Rappelons que la traduction consiste à mettre à la disposition d'un lecteur, par définition ignorant de la langue de départ, un texte rapportant le plus complètement et le 
plus clairement possible la pensée d'un auteur étranger. Elle constitue donc le seul critère que l'on puisse avoir pour juger de l'équivalence de deux situations exprimées dans deux langues différentes. Appeler traduction un texte que l'on ne peut lire qu'avec l'original allemand en regard et un dictionnaire, comme le conseille François Vezin pour son Übersetzung de Heidegger, c'est offrir une boîte de cirage à un chauve... Pourquoi dans ces conditions traduire? Mieux vaut suivre l'exemple de Jean Beaufret, qui recommandait à ses étudiants de lire Heidegger dans le texte. Est-il en effet rien de plus absurde, irréaliste et inutile que de travailler pendant des années à une traduction qui se définit elle-même comme n'en étant pas une ?

André Bourguignon nous dit que la nouvelle traduction des OCF doit nous permettre de retrouver Freud - attention, pas celui que l'on connaît (mal) à travers les précédentes traductions, mais le vrai Freud, le $U r$ Freud. Vouloir retrouver ce Freud, par opposition à un Freud «francisé», c'est demander aux lecteurs français d'apprendre l'allemand, donc nier l'existence même de la traduction. (Exit le traducteur.) Qu'est-ce en effet qu'une traduction pour un lecteur français sinon une francisation? Cette francisation, c'est-à-dire la transmission d'un texte étranger, est par essence un choix certes arbitraire - dont le traducteur doit rester le seul maître. Il a le droit le plus strict d'utiliser toutes les terminologies et tous les dictionnaires qu'il voudra, à condition qu'il ne se complaise pas dans une «étymologisation» stérile.

N'oublions pas en effet que le mot ne se réduit pas à la somme de ses composantes $($ Sehn + sucht $\geq$ Sehnsucht). Prenons la notion de bien-être. Apparemment, l'espagnol bienestar, le français bien-être et l'anglais welfare sont étymologiquement formés de la même façon. Néanmoins, si l'on examine de plus près le signifiant anglais well-fare (bien se porter), on constate que l'étymologie ne nous est d'aucune aide en tant que traducteur, puisque ce mot a pris en anglais moderne, et surtout en américain, une connotation sociale très prononcée : on traduira dans un cas welfare state par «providence», dans un autre on welfare par «aide sociale». D'autre part, si l'on considère bienestar, construit apparemment sur un modèle plus proche de «bien-être», deux remarques s'imposent. Premièrement, le verbe être a deux équivalents en espagnol, ser et estar, correspondant grosso modo l'un à l'essence, l'autre à l'accident - on peut ainsi être amené à traduire estar par se trouver (faudrait-il alors, en bon freudologue, parler d'un «bien-setrouver» ?). Deuxièmement, la présence à côté de bienestar de son contraire malestar et l'absence d'un équivalent mal-être en français font que le terme ne peut pas recouvrir le même champ sémantique que bien-être. Cet exemple montre qu'il est impossible de faire correspondre mathématiquement les mots de deux langues. Le sens d'un mot se définit par la somme de ses emplois (c'est ce que Saussure appelle la «valeur») et par sa place dans une phrase. Se placer au niveau du seul mot, c'est passer à côté du problème. On peut toujours traduire des termes isolés, mais en mettant bout à bout tous les dictionnaires de psychanalyse, on n'arrivera jamais à la pensée de Freud. En effet, le mot s'inscrit dans une phrase et ne prend son sens que par contact avec les autres mots.

Ainsi, MM. Laplanche et Bourguignon ont le droit de choisir de traduire Wunsch par «souhait» au lieu de «désir», s'ils veulent par là exprimer un type d'attirance ne se portant pas sur une personne. Cela ne les autorise pas à obliger leurs traducteurs à substituer automatiquement «souhait» à Wunsch. Il est évident que souhait formateur de rêve ne fait pas sens en français - a-t-on jamais pris un souhait pour une réalité ? De plus, comment ne pas plaindre les «nouveaux traducteurs» (comme on dit nouvelle cuisine) obligés de trimballer dans leur musette 5000 mots français à greffer sur des phrases fragiles, union contre nature dont les fruits monstrueux finiront par tuer l'arbre 
qui les porte. Ils nous font penser à ces vacanciers nostalgiques qui insistent pour porter à Paris les oripeaux bariolés achetés sous les tropiques.

Or, c'est sur cet exotisme voulu que repose la nouvelle traduction de Freud. Il fut un temps où le traducteur qui n'avait pas lu Laplanche pensait naïvement qu'on traduisait mieux en lisant une page puis en refermant le livre. On a du mal à croire qu'avec des méthodes aussi primitives cet individu ait réussi à traduire Stuart Mill et Charcot. Sigmund Freud - car c'était lui - essayait de penser les textes en allemand. C'est précisément ce que lui reproche Jean Laplanche : «[...] en quoi évidemment Freud était foncièrement influencé par la culture française, [sic] et si éloigné, dans cette approche globale du texte [...] de sa propre méthode analytique ${ }^{5}$ !» Lecomte de Lisle, lui, n'avait pas lu Laplanche, et pourtant n'a-t-il pas traduit Peleiados Akhilleus (Achille fils de Pélée) par «le Peleiade Akhilleus»? De même, pour faire russe, on nous inflige des Gracieuse Maria Alexandrovna ou des Honoré Ivan Petrovitch aussi indigestes que des blinis à la crème de marrons. Pourquoi pas Maria ou Ivan - ou même rien du tout ? Et les archines, et autres verstes et pas mûres ! Doubler Laurel et Hardy en français avec un accent américain, c'est une idée absurde mais ça fait rire. Faire parler Freud en français avec un accent allemand est tout aussi aberrant mais beaucoup moins drôle. C'est d'autant moins drôle que les responsables de la traduction des OCF s'appuient sur des justifications théoriques qui n'ont de sérieux que leur apparence. Bizarrement, depuis quelques années, on voit fleurir des théories «nonsensiques» selon lesquelles une traduction digne de ce nom doit sentir la traduction, comme un bon camembert le camembert. Antoine Berman dit-il autre chose quand il appelle mauvaise traduction celle «qui, généralement sous couvert de transmissibilité, opère une négation systématique de l'étrangeté de l'cuvre étrangère ${ }{ }_{»}$ ? Chouraqui va encore plus loin : sous prétexte que les auteurs du Nouveau Testament écrit en grec «étaient plongés dans l'univers langagier de l'hébreu et de l'araméen [...] il retraduit le grec dans la visée de l'hébreu?». Le père fondateur de cette littéralité n'est autre que Martin Heidegger. Il définit une méthode qui consiste à «écouter en grec» un texte de Parménide écrit il y a plus de deux mille ans. On peut se demander comment un philosophe allemand $\mathrm{du} \mathrm{XX}^{\mathrm{e}}$ siècle a réussi à retrouver ce que Parménide entendait par «l'être de l'étant». Encore la démarche de Heidegger se justifie-t-elle en ce sens qu'il cherche à remonter aux racines de la pensée; mais dans le cas de la traduction de Freud, l'archéologie des mots n'est pas seulement présomptueuse : elle est vaine. Par définition, le penseur construit sa pensée et son vocabulaire à mesure qu'il écrit. Comme le dit Merleau-Ponty: «Nous commençons à lire le philosophe en donnant aux mots qu'il emploie leur sens "commun", et, peu à peu, par un renversement d'abord insensible, sa parole maîtrise son langage, et c'est l'emploi qu'il en fait qui finit par les affecter d'une signification nouvelle et propre à lui [...]. Car désormais les démarches préparatoires de l'expression - les premières pages du livre - [les premiers écrits de Freud] sont reprises dans le sens final de l'ensemble et se donnent d'emblée comme dérivées de ce sens, maintenant installé dans la culture ${ }^{8}$.»

On peut sans trop de risque appliquer cette réflexion à la traduction, qui «installe» un sens dans la culture, même si c'est en dépit du bon sens... Il est des contresens sains qui supplantent avantageusement le «bon» sens. Citons «paradis fiscal», à partir de l'anglais tax haven (havre fiscal) confondu avec tax heaven (paradis). Il est des contresens insanes, voire freudlatés...

Encadré de deux gendarmes, Jérôme pénètre dans la salle d'audience. Le jury, entièrement composé de traductologues et déployé sur trois rangs de gradins, est plongé dans une profonde méditation que l'arrivée du prévenu ne semble pas troubler. Jérôme 
prend place dans le box des accusés. Le juge entre. L'assistance se lève. On lit les chefs d'inculpation - trois au total.

- Jérôme Lherer, vous êtes accusé d'avoir commis une faute rédhibitoire et, ce faisant, d'avoir dénaturé Souffler n' est pas jouer, le livre de Cárcel Mayor dont vous avez assuré la traduction française.

- Vous êtes en outre accusé d'avoir camouflé votre délit en n'en avisant pas votre éditeur, ainsi que l'exige la loi.

- Enfin, vous êtes accusé d'avoir essayé de racheter à bas prix tous les exemplaires de l'ouvrage précité, dans une vaine tentative de dissimulation du corpus delicti.

Jérôme sent l'amertume l'envahir. Comment ne serait-il pas amer? Dire qu'il a passé des heures à se mettre dans la peau de l'auteur, passé des heures à gommer tous les traits qui risquaient de le trahir, des heures à prêter sa voix, ses mots, son cœur enfin à un inconnu qui l'oblige à être, en même temps que son ombre, l'ombre de lui-même...

- Avez-vous quelque chose à dire pour votre défense ?

Jérôme sort de sa rêverie... Tous les regards sont tournés vers lui. C'est sa dernière chance de se réhabiliter aux yeux de la société. Il avale sa salive, ouvre la bouche... Aucun mot ne sort. La sueur perle sur son front à présent aussi buriné que celui de Cárcel Mayor...

- Qui ne dit mot consent, assène le juge.

Le jury délibère. Jérôme attend, le cour serré. Son calvaire ne dure heureusement pas longtemps: il aura fallu 1 minute 35 secondes aux traductologues pour se mettre d'accord. Au moment où le juge se lève pour prononcer le verdict, le temps semble se figer...

- Jérôme Lherer...

Soudain une sonnerie stridente résonne. L'assistance conserve un calme imperturbable. Une deuxième, puis une troisième sonnerie. Le juge continue de lire le verdict... Le feu ! pense Jérôme. Il s'est levé d'un bond de son siège. Vite, il faut fuir! Deux paires de bras musclés l'obligent à se rasseoir. Et le juge qui poursuit sa lecture d'une voix monocorde... Dans un suprême effort, Jérôme parvient à se dégager de l'étreinte de ses geôliers. Juste à temps pour s'emparer du téléphone, qui gît par bonheur au pied de son lit... Aires..

- Monsieur Lherer, ne quittez pas, je vous passe une communication de Buenos

Paul Valéry nous fournit le meilleur remède aux angoisses des traducteurs: «Traduire, c'est reconstruire d'aussi près que possible l'effet d'une certaine cause au moyen d'une autre cause ${ }^{9} . \gg$ Refuser de changer la cause, c'est dénaturer l'effet ou le supprimer. Un chauffeur d'autobus new-yorkais dit gentiment «Watch your step» à chaque voyageur qui descend. Imaginons un groupe de touristes français du troisième âge, accompagné d'un guide - interprète bermanien. Il ne peut pas traduire par Attention à la marche, ce qui serait «ethnocentrique». Il va penser la remarque en anglais, aboutissant à un énoncé du style Surveillez votre marche! (Watch-your-step). La vieille dame de la Creuse, déjà fatiguée et affamée (elle a refusé tous les hamburgers et s'obstine à réclamer un pâtié à la treffés ${ }^{10}$ ), s'approche de la porte en se demandant ce que veut dire Surveillez votre marche. Elle se souvient que dans sa jeunesse on disait ça aux filles de mauvaise conduite. Elle sourit, descend et se casse le col du fémur.

Vouloir préserver la cause aux dépens de l'effet, c'est réduire le texte à une succession de mots, réduire Freud à une suite de fiches terminologiques, que l'on pourra bientôt consulter, pour une pièce de dix francs, dans les supermarchés et les stations de métro, avec en prime l'horoscope du jour et la description du compagnon idéal pour la 
semaine. C'est mettre Freud au rang des auteurs sacrés. Le texte sacré, en effet, c'est celui qu'on ne doit pas traduire, comme le Coran aux yeux des intégristes.

Vouloir à tout prix conserver un texte dans la langue originale, c'est sacrifier l'effet à la cause : c'est ôter à ce texte toute sa force de conviction pour le réduire à l'incantation pure, c'est-à-dire à la magie du mot et du son. Attitude compréhensible bien qu'autodestructrice chez un ayatollah, puisqu'elle freine le prosélytisme, à la rigueur justifiable en poésie (le Corbeau aurait pu croasser Nevermore en français), mais carrément absurde pour un texte à vocation pédagogique et scientifique.

Nous ne pouvons pas croire que les freudologues cherchent à tirer à eux le fil d'Ariane pour s'en faire une pelote, voire un bas de laine. Mais alors, pourquoi considèrent-ils le texte de Freud comme intouchable ? Il s'agit à nos yeux d'un véritable refus de traduire, c'est-à-dire d'assimiler l'étranger. Ce n'est certainement pas un hasard si cette démarche «anti-ethnocentrique» s'inscrit dans une époque dominée par la décolonisation. On dirait que nos traductologues, en critiquant la traduction «à la française», cherchent à expier les péchés du colonialisme français dont pourtant ils ne sont en rien responsables. La traduction, faut-il le dire, n'a rien à voir avec la colonisation. Elle a existé bien avant et existera longtemps après. Les ethnocentristes ne sont pas ceux qui traduisent le Coran en français, mais ceux qui obligent les étrangers à apprendre l'arabe pour le lire. La démarche des traductologues est à replacer dans le cadre plus large du complexe d'infériorité vis-à-vis des cultures étrangères qui, par définition, échapperaient (excision comprise) à notre «bon sens» occidental.

- Allô, Jérôme, tu m'entends?

-Allo. Tu vas bien?

- Très bien. Je n'ai pas beaucoup de temps. Je pars en excursion dans dix minutes. Mais avant je voulais te lire quelque chose qui te concerne. C'est paru dans la Pampa Libre et c'est la réceptionniste de l'hôtel qui a bien voulu me le traduire. Je te le livre tel quel. «Le livre de Cárcel Mayor, qui n'a recevu pas la gloire dans son pays propre ni en aucun autre de l'Amérique du Sud est faisant l'objet d'une excitation clamoureuse en France. C'est possiblement la traduccion de Jérôme Lherer qui a désenchainé une telle excitation car son texte a une calité maximalement bonne de celui de Cárcel Mayor...»

Le traducteur est le témoin privilégié de l'insignifiance du discours contemporain, le lieu géométrique de l'absurdité du langage. La légende rapporte l'histoire de cet interprète, au temps de la SDN, qui, prenant fait et cause pour un petit pays opprimé, avait transformé le discours terne du délégué en plaidoyer émouvant. Le délégué ayant eu la bêtise de lui reprocher après la séance de n'avoir pas traduit fidèlement ce qu'il avait dit, s'attira cette réponse sublime: «Monsieur, c'est ce que vous auriez dû dire.» Tolérerait-on aujourd'hui cette insolence qui prétend donner une âme à des propos creux ? Malheureusement, la question ne se pose pas: les inconditionnels du week-end congresseux n'ont plus peur de parler pour ne rien dire. On ne demande pas au traducteur d'être intelligent. Si même on pouvait lui couper la langue et le transformer en gardien muet du sérail où les nouveaux sultans caressent les platitudes internationales !

On pourrait même se passer de ses services puisque nous vivons la civilisation des grands mots. Et c'est peut-être ici que se situe le centre de gravité (au sens lourd) de la bêtise contemporaine. Elle trouve sa meilleure forme d'expression dans le jargon. Qu'estce qu'un jargon sinon un enchaînement de mots reliés par une syntaxe rudimentaire? Pourquoi se donner la peine de structurer sa pensée ? Il suffit de prononcer les mots «2 produits en 1», «technologie de pointe», «événement médiatique», «vie active» (par opposition à l'école, comme si l'enfance n'était pas l'âge le plus actif de l'existence) pour faire saliver les vieux chiens asthmatiques de la société sciento-capitaliste. Pourquoi un chercheur français ferait-il traduire sa communication puisque de toute façon son texte 
consiste à enfiler bout à bout des termes qu'il connaît en anglais ? Ses collègues le comprendront puisqu'ils savent de quoi il parle (normalement, quand on sait de quoi un article parle, on n'a pas envie de le lire... mais c'est peut-être ça la communication).

Menacé par l'objectivation croissante du langage, le traducteur croit trouver un refuge dans l'objectivité. De peur d'être supplanté par l'ordinateur, il se transforme en machine à traduire. Or il se trouve que l'objectivité en traduction a pour fonction de transmettre une subjectivité - situation par essence insoluble car on ne peut rendre objectivement la subjectivité d'un autre sans passer par la sienne propre. Le traducteur ne peut trouver le salut qu'en assumant sa subjectivité, en refusant de se laisser enfermer dans les bêtises d'un autre. Ce document que j'ai devant les yeux est-il vraiment plein de pièges, de faux-amis embusqués entre les lignes? Ou les fautes sont-elles de moi, l'ambiguïté ajoutée à la lecture par cette horreur du vide sémantique que j'éprouve devant un texte aussi insignifiant, ce besoin d'y transfuser une goutte de sens? Et si tel est le cas, pourquoi cette déformation que j'introduis, image réfractée et réfractaire du texte original, ne le rendrait-elle pas plus «droit» qu'il était ? Pourquoi mon mensonge serait-il moins vrai que la vérité d'un imbécile ? Comme le dit Jakobson: "Traducteur de quels messages? Traître à quelles valeurs ${ }^{11}$ ?»

Et si je laissais parler en moi ce Grec que j'étais bien avant de lire Parménide? Pourquoi m'efforcerais-je, par une archéologie besogneuse, de reconstituer cette pensée qui, de toute façon, m'habite? «Nous aussi portons en nous une invisible et inconsciente grécité ${ }^{12} . »$ Je ne pourrai jamais penser uniquement en français. Ne pas faire confiance à cet «étranger» en moi serait la pire des bêtises...

Et si j'oubliais un peu la peur de la faute, du péché originel ? Et si je traduisais pour me faire plaisir ? Combien de fleurs cueillies en vain, combien de coquillages ramassés sur la plage, combien de cadeaux ignorés a-t-il fallu pour que j'en arrive à me méfier de mes interprétations? Combien de contresens faudra-t-il pour que j'ose enfin devenir traducteur? «Nous devons accoutumer nos prochains graduellement, précautionneusement, à ce fait que nous sommes vivants ${ }^{13}$.»

Et Jérôme de jurer : plus jamais solique en ma coquitude 14.

Notes

1. THOMAS, Henri (1956): La Nuit de Londres, Paris, Gallimard, 148 p.

2. Groupe révolutionnaire panaméricaniste, boycotté pour des raisons obscures par les disquaires français. Nos lecteurs pourront néanmoins se procurer le disque en question en s'adressant au diffuseur parisien du groupe, place de la Porte dc Montreuil (angle boul. Davout - av. de la Porte de Montreuil) le dimanche uniquement, de 14 à 18 heures.

3. Nous donnons ci-dessous le texte des 2 strophes concernées. Comme on le voit, Baudelaire déplace certains éléments de l'anglais.

Art is long and Time is fleeting,

And our hearts, though stout and brave,

Still, like muffled drums, are beating

Funeral marches to the grave.

$$
\text { A Psalm of Life }
$$

Full many gem of purest ray serene

The dark unfathom'd caves of ocean bear:

Full many a flower is born to blush unseen.

And waste its sweetness on the desert air

Elegy Written on a Country Churchyard

4. Propos tenus par André Bourguignon au cours de l'émission «Libre et Change», mai 1988.

5. LAPLANCHE, Jean (1988): «Le mur et l'arcade», Nouvelle Revue de psychanalyse, vol. XXXVII, p. 99.

6. BERMAN, Antoine (1984): L'Épreuve de l'Étranger, Paris, Gallimard, p. 17

7. LAPLANCHE, Jean, op. cit., p. 104

8. MERLEAU-PONTY, Maurice (1953): «Sur la phénoménologie du langage», Éloge de la philosophie, Paris, Gallimard, pp. 98-99. 
9. VALÉRY, Paul (1941): Préface, Cantiques spirituels de Saint Jean de la Croix, traduits par le père Cyprien.

10. Expression creusoise signifiant «pâté aux pommes de terre». Mme Tallot, boulangère à Saint-Dizier-lesDomaines, canton de Chatelus Mavaleix (Creuse), offrira gracieusement à nos lecteurs ce régal du cru.

11. JAKOBSON, Roman (1963): Éléments de linguistique générale, trad. N. Ruwet, Paris, Éditions de Minuit, p. 86.

12. LACARRIÈRE, Jacques (1976): L'Été grec, Paris, Plon, p. 385.

13. KOSZTOLANYI, Dezsö (1985): «La Disparition», Le traducteur cleptomane et autres histoires, trad. M. Régnaut et P. Adam, Paris, Alinéa, p. 65.

14. Inspiré de la superbe traduction de Philippe Lavergne pour Loonely in ne loneness, James Joyce, Finnegan's Wake. 